\title{
Performance Analysis of Portfolio Optimisation Strategies: Evidence from the Exchange Market
}

\author{
Jason Narsoo ${ }^{1}$ \\ ${ }^{1}$ Department of Economics and Statistics, Faculty of Social Studies and Humanities, University of Mauritius, \\ Réduit, Mauritius \\ Correspondence: Jason Narsoo, Department of Economics and Statistics, Faculty of Social Studies and \\ Humanities, University of Mauritius, Réduit, Mauritius. Tel: 230-403-7948. E-mail: j.narsoo@ uom.ac.mu
}

Received: May 12, 2016

doi:10.5539/ijef.v9n6p124
Accepted: June 24, 2016

Online Published: May 15, 2017

\begin{abstract}
Portfolio allocation is embedded in many decisional tasks for ensuring best returns under the constraint of minimising risk. In this paper, we implement several strategies in order to generate a holistic assessment of portfolio evaluation. The study analyses the performance of an extended framework of the classical tangency and targeted portfolio strategies. The extension is essentially the use of the skewed student-t distribution for the individual assets' log-return. Our investigation is based on 15 currencies with US dollar as the base currency for the period spanning from 1999 to 2015. A comparative performance analysis between the portfolio optimization strategies is undertaken on the basis of various performance measures, namely the portfolio expected return, standard deviation, Beta coefficient, Sharpe Ratio, Jensen's Alpha, Treynor ratio and Roy ratio. The portfolio VaR being perceived as one of the core metrics for risk management is also computed. It is actually proxied by 5 VaR estimates - the parametric Gaussian, the equally-weighted historical VaR, the bootstrapping historical VaR, the Monte-Carlo simulation VaR and the parametric GHD VaR. The results show that both tangency portfolios, with the Gaussian or the skewed student-t distribution perform best, particularly on the basis of highest Sharpe reward-to-variability ratio and lowest Value-at-Risk.
\end{abstract}

Keywords: portfolio optimization, exchange rates, performance measures, value-at-risk

\section{Introduction}

The generic principle embedded in portfolio analysis relates to the notion of "do not put all your eggs in one basket". The basic tenet of such a statement is that if the basket falls, all the eggs are broken. In the same vein, fund managers worldwide resort towards portfolio allocation in view of ensuring the best returns being optimized under various economic conditions subject to adherence to the stipulated risk-profile of the clients. The theory of portfolio analysis is now embedded not only for securities analysis but also for other important decisional tasks. For instance, credit portfolio analysis is now an inherent feature of any commercial bank in view of harnessing the maximum returns feasible.

The current study undertakes a comprehensive and rigorous analysis of a portfolio of 15 currencies with US dollar as the base currency for the period spanning from 1999 to 2015. We construct various strategies, namely equal allocation, single index model with short sales, single index model without short sales, tangency portfolio based on normal distribution, tangency portfolio based on skewed student-t distribution, targeted portfolio return based on normal distribution and targeted portfolio return based on skewed student-t distribution. In essence, seven strategies are considered to generate a holistic assessment of portfolio evaluation. Currencies constitute the focus of our study as data is freely available on yahoo finance, let alone the fact that the foreign exchange market is the only market which operates 24 hours over 24 hours throughout the whole week. Consequently, liquidity bias is less susceptible to buffet in our results relative to the equity or commodity markets.

The primary aim of the paper is to gain insight as to which strategy generates the best risk-returns tradeoff. However, care may be exercised in the sense that a specific strategy may trail behind the highest return subject to certain impediment such as higher cost. This is akin to hedging in the field of finance whereby GARCH model tends to generate better out-of-sample portfolio performance but at the cost of having more portfolio reshuffling effects. This can also be captured out via active portfolio management which undeniably unleashes higher costs relative to passive portfolio management strategy. 
The rest of this paper is organized as follows. Section two focuses on the literature review and section three deals with the data and methodology. Section four discusses on the results obtained while section five concludes.

\section{Literature Review}

A major breakthrough pertaining to portfolio asset allocation occurred in 1952 ensuing the seminal work of Markowitz. At its core development level of the theory of portfolio diversification, Markowitz (1952) assumed free market forces without hindering effects of taxes or transaction costs, infinitely divisible assets to be traded, no room for short-sales and negative proportions of assets to be incorporated in the portfolio. Most importantly, the crux of portfolio analysis relied heavily on the first two moments of asset returns, namely risk and return under a mean-variance analysis framework. Under such a framework, based on a given expected return, the whole objective is geared towards minimization of the variance.

Sharpe (1964) came forward with the term Capital Asset Pricing Model (CAPM) to generate proper demarcation line between diversifiable risk and systematic risk. The gist of such a framework is that systematic risk is non-diversifiable by leveraging on more and more securities in a portfolio. Consequently, investors need to be sufficiently rewarded for shouldering systematic risk. The next evolution in the field came from the work of Borch, Hester and Tobin (1969) who incorporated a risk-free asset in the Markowitz framework to come forward with the Separation theorem concept. The latter argues that in the presence of a risk-free asset, the optimal risky portfolio can be sieved out without knowledge of the investors' risk preferences.

In 1976, Ross pushed forward the portfolio theory framework via the Arbitrage Pricing Theory. In a nutshell, the distinctive feature of the Arbitrage Pricing Theory (APT) relative to CAPM refers to the fact that the former considers a certain number of macroeconomic variables while the latter only factors in the market returns. Both APT and CAPM presume linear associations to the forces which drive asset returns.

Throughout the years, portfolio theory underwent major changes. For instance, the CAPM is now modelled under the Generalised Autogressive Conditional Heteroscedasticity (GARCH) approach based on the existence of volatility clustering (symmetric GARCH) and leverage effects (asymmetric GARCH). Studies stretching beyond conventional first two moments have also been considered such as Sereda et al. (2010). Advanced technique from mathematical physics was also considered such as asset selection filters based on Random Matrix Theory as performed by Daly et al. (2008). Another important evolution came when utility functions were incorporated when modelling the portfolio asset returns under Von Neumann-Morgenstern's (1953) Expected Utility Hypothesis. Black and Litterman (1992) gave due consideration to investors' opinions when modelling asset returns. Today, the use of priors is now deemed as an important advancement in the field of finance under the Bayesian approach to modelling.

With time, the main advancements in the field of portfolio theory were more of computational design in lieu of theoretical advancements, mainly propelled by the use of high quality technology. Studies such as Parpas and Rustem (2007) and Troha (2011) used stochastic programming and simulations. In the case that random variables manifest in the problem statement, recourse is made towards stochastic optimization methods. Crama and Schyns (2003) used simulated annealing based technique to model portfolio selection. Hochreiter (2008) resorted to evolutionary stochastic portfolio optimization using genetic algorithm. Mishra et al. (2009) implemented a multi-objective particle swarm optimization method for the portfolio optimization problem. Studies have also incorporated neural networks approach to portfolio analysis such as those of Zimmermann et al. (2001) and Steiner and Wittkemper (1997).

The choice of performance indicators for portfolio evaluation is of fundamental importance. Caporin and Lisi (2011) defined four types of performance indicators among which we have absolute performance measures and relative performance measures. Absolute performance evaluation is performed using the indicators in isolation. On the other hand, relative performance measures help investors in analyzing their portfolios as compared to market benchmarks or to other strategies, which is a more sensible assessment approach. Generally, the comparative analyses are performed on the basis of two dimensions namely the expected return and the risk. Before the 1960s, investors were overlooking the risk component in the composition of their portfolios as their main focus was mistakenly based on the returns. However, over time investors dedicated more and more importance to portfolio risk assessment. The most famous risk indicator is the volatility of returns. Though it proved interesting for comparative analysis, the volatility does not capture the total risk picture of returns, especially for portfolios exhibiting skewed return distributions. The Value-at-Risk (VaR) actually overcomes this major limitation, its focus being on downside risk and potential losses. In other words, VaR captures the tail end of the returns to the down side. In the paper of Koh and Lee (2011), the portfolio assessment is performed using other sets of performance measurement tools which are also known as the risk-adjusted performance measures. 
These indicators are the Sharpe ratio, the Jensen's alpha, the Treynor ratio and the Roy ratio, which are merely a combination of risk and return performance.

Basically, the literature on portfolio theory is expected to develop more on the computational side in lieu of the theoretical side. Nonetheless, the main focus lies in the trade-off between the costs of using a highly sophisticated technique and the benefits emanating from the use of that specific technique.

\section{Methodology}

This paper analyses the performance of different portfolio optimization strategies. The investigation is based on 15 currencies with US dollar as the base currency for the period spanning from 1999 to 2015. Using currency data eventually reduces the susceptibility of having liquidity bias buffeting in our results. The data is freely downloaded on yahoo finance.

\subsection{Asset Allocation Strategies}

This paper constructs seven portfolio strategies namely, equal allocation, single index model with short sales, single index model without short sales, tangency portfolio based on normal distribution, tangency portfolio based on skewed student-t distribution, targeted portfolio return based on normal distribution and targeted portfolio return based on skewed student-t distribution.

\subsubsection{Equal Allocation}

The equal allocation is also referred to as a "naïve strategy" as it is basically an equally weighted portfolio. This passive allocation strategy will be used as a benchmark for our active optimization strategies.

\subsubsection{Single Index Model}

The single index model is a computationally simplified version of the Markowitz's model which was developed by Sharpe (1963). It is formulated as

$$
R_{i t}=\alpha_{i}+\beta_{i} R_{M T}+\epsilon_{i t}
$$

where $R_{i t}$ denotes the return on asset $i, R_{M T}$ denotes the market index return and $\epsilon_{i t}$ denotes the specific return on asset $i$. The parameters $\alpha_{i}$ and $\beta_{i}$ are to be determined empirically by regressing asset returns against market returns.

The determination of optimal portfolios makes use of the simplified correlation representation model to establish an optimal ranking of the portfolio potential components. A threshold is then determined in the list such that only those securities above that threshold are retained to compose the portfolio. In this study, the Treynor Ratio (TR) is used to rank the assets from the highest ratio to the lowest. Indeed, the higher the TR, the more desirable it is to include the asset in the portfolio. The Treynor Ratio for asset $i$ is calculated as:

$$
T R_{i}=\frac{E\left(R_{i}\right)-R_{f}}{\beta_{i}}
$$

In this study, the risk-free interest rate $R_{f}$ is assumed to be $0 \%$, since this study investigates portfolio allocation in the exchange market.

The threshold value $C^{*}$ is calculated using an iterative procedure. In fact, a value $C_{i}$ for a portfolio comprising $j$ securities is calculated each time a security is included in the portfolio. It is computed using the expression

$$
C_{i}=\frac{\sigma_{M}^{2} \sum_{j=1}^{i} \frac{\left(E\left(R_{i}\right)-R_{f}\right) \beta_{j}}{\operatorname{var}\left(\epsilon_{j}\right)}}{1+\sigma_{M}^{2} \sum_{j=1}^{i} \frac{\beta_{j}^{2}}{\operatorname{var}\left(\epsilon_{j}\right)}}
$$

where $\sigma_{M}^{2}$ is the variance of market returns. Securities from the list are no more added to the portfolio when the corresponding TR is lower than the $C_{i}$ calculated for the previous portfolio.

The optimal weights $x_{i}$ for the single index model are determined by:

$$
x_{i}=\frac{z_{i}}{\sum_{i=1}^{N} z_{i}}
$$

where $N$ is the number of components in the portfolio and the parameter $Z_{i}$ is obtained by the expression:

$$
Z_{i}=\frac{\beta_{i}\left(T R_{i}-C^{*}\right)}{\operatorname{var}\left(\epsilon_{i}\right)}
$$


In this study, the single-index model is implemented using two distinct approaches:

(1) when short-sales is not allowed, $C^{*}=\max \left(C_{i}\right)$

(2) when short-sales is allowed, $C^{*}=$ last $C_{i}$ in the portfolio

\subsubsection{Tangency Portfolio}

The tangency portfolio is defined as being the composition of securities which maximizes the corresponding Sharpe Ratio (SR). The portfolio Sharpe Ratio $S R_{p}$ is calculated as:

$$
S R_{p}=\frac{E\left(R_{p}\right)-R_{f}}{\sigma\left(R_{p}\right)}
$$

This ratio is often referred to as the reward-to-variability ratio. The strategy is therefore defined by a maximization problem over $S R_{p}$.

This study implements the strategy using two distinct underlying distributions namely the normal distribution and the skewed student-t distribution.

\subsubsection{Targeted portfolio return}

The targeted portfolio return works in the Markowitz's mean-variance framework where the objective is to minimize the portfolio risk constrained to a target return, which is set to $0.5 \%$ in this study. The optimization problem is therefore formulated as follows:

$$
\begin{array}{r}
\operatorname{Min} \sigma\left(R_{p}\right)=\sqrt{\sum_{i} \sum_{j} \omega_{i} \omega_{j} \operatorname{Cov}_{i, j}}=\sqrt{\boldsymbol{\omega}^{T} \boldsymbol{\Sigma} \boldsymbol{\omega}} \\
\text { subject to }\left\{\begin{array}{c}
\sum_{i} \omega_{i} E\left(R_{i}\right)=\boldsymbol{\omega}^{T} \boldsymbol{\mu}=0.5 \% \\
\sum_{i} \omega_{i}=1
\end{array}\right.
\end{array}
$$

where $\omega_{i}$ denotes the allocation weight to asset $i$ and $\operatorname{Cov}_{i, j}$ is the covariance between returns of assets $i$ and $j$. In matrix representation, $\omega$ is the vector of allocation weights and $\Sigma$ denotes the variance-covariance matrix of all the securities.

It is assumed that short-selling is possible in this strategy. As for the tangency portfolio strategy, the normal distribution and the skewed student-t distribution are assumed.

\subsection{Performance Measures}

The comparative analysis between the different optimal portfolio strategies and the passive asset allocation

\begin{tabular}{|c|c|c|}
\hline Portfolio Performance Measures & \multicolumn{2}{|l|}{ Definition } \\
\hline 1. Expected return & $E\left(R_{p}\right)=\sum_{i} \omega_{i} E\left(R_{i}\right)=\boldsymbol{\omega}^{\boldsymbol{T}} \boldsymbol{\mu}$ & (8) \\
\hline 2. Standard deviation & $\sigma\left(R_{p}\right)=\sqrt{\omega^{T} \boldsymbol{\Sigma} \boldsymbol{\omega}}$ & (9) \\
\hline \multirow[t]{3}{*}{ 3. Beta coefficient } & $\beta_{p}=\frac{\operatorname{Cov}_{p, M}}{\sigma_{M}^{2}}$ & (10) \\
\hline & where & \\
\hline & $\begin{array}{l}\text { - } \operatorname{Cov}_{p, M} \text { is the covariance between the portfolio retu } \\
\text { - } \quad \sigma_{M}^{2} \text { is the variance of the market index returns }\end{array}$ & \\
\hline 4. Sharpe ratio & $S R_{p}=\frac{E\left(R_{p}\right)-R_{f}}{\sigma\left(R_{p}\right)}$ & (11) \\
\hline \multirow[t]{2}{*}{ 5. Jensen's alpha } & $\begin{array}{l}\text { It is defined as the difference between the portfolio return and } \\
\text { for the portfolio, hence given by: }\end{array}$ & eturn \\
\hline & $\alpha_{\text {Jensen }}=R_{p}-\left[R_{f}+\beta_{p}\left(R_{M}-R_{f}\right)\right]$ & (12) \\
\hline
\end{tabular}
strategy is performed using different performance metrics which are presented in the table below.

Table 1. Definition of the portfolio performance measures 
6. Treynor ratio

7. Roy ratio

8. Value-at-Risk (VaR)

$$
T R_{p}=\frac{E\left(R_{p}\right)-R_{f}}{\beta_{p}}
$$

$$
R R_{p}=\frac{E\left(R_{p}\right)-E\left(R_{M}\right)}{\sigma\left(R_{p}\right)}
$$

where $R_{M}$ is the expected return of the returns of the market index.

It is defined as the maximum loss that an investor can expect to incur given a specified time horizon and a confidence level $\alpha \%$. In this study, five different estimates for the VaR are calculated namely :

- the parametric Gaussian

- the equally-weighted historical VaR

- $\quad$ the bootstrapping historical VaR

- $\quad$ the Monte-Carlo simulation VaR

- the parametric GHD VaR

\section{Results}

\subsection{Preliminary Analyses}

The Shapiro-Wilks and Jarque-Bera normality tests reveal that the individual currency log-returns are not normally distributed except for USD/EUR, USD/JPY and USD/SEK currencies. The corresponding normality hypothesis being not rejected for at least one of the statistical tests. The observations are confirmed by the boxplots and the histogram graphs in Figure 1 and 2 respectively. The currencies which failed the normality tests display a distribution with extreme outliers, hence showing a skewed rather than a symmetric distribution shape. This observation hence supports our methodology to employ a skewed student-t log-return distribution within the strategies' framework.

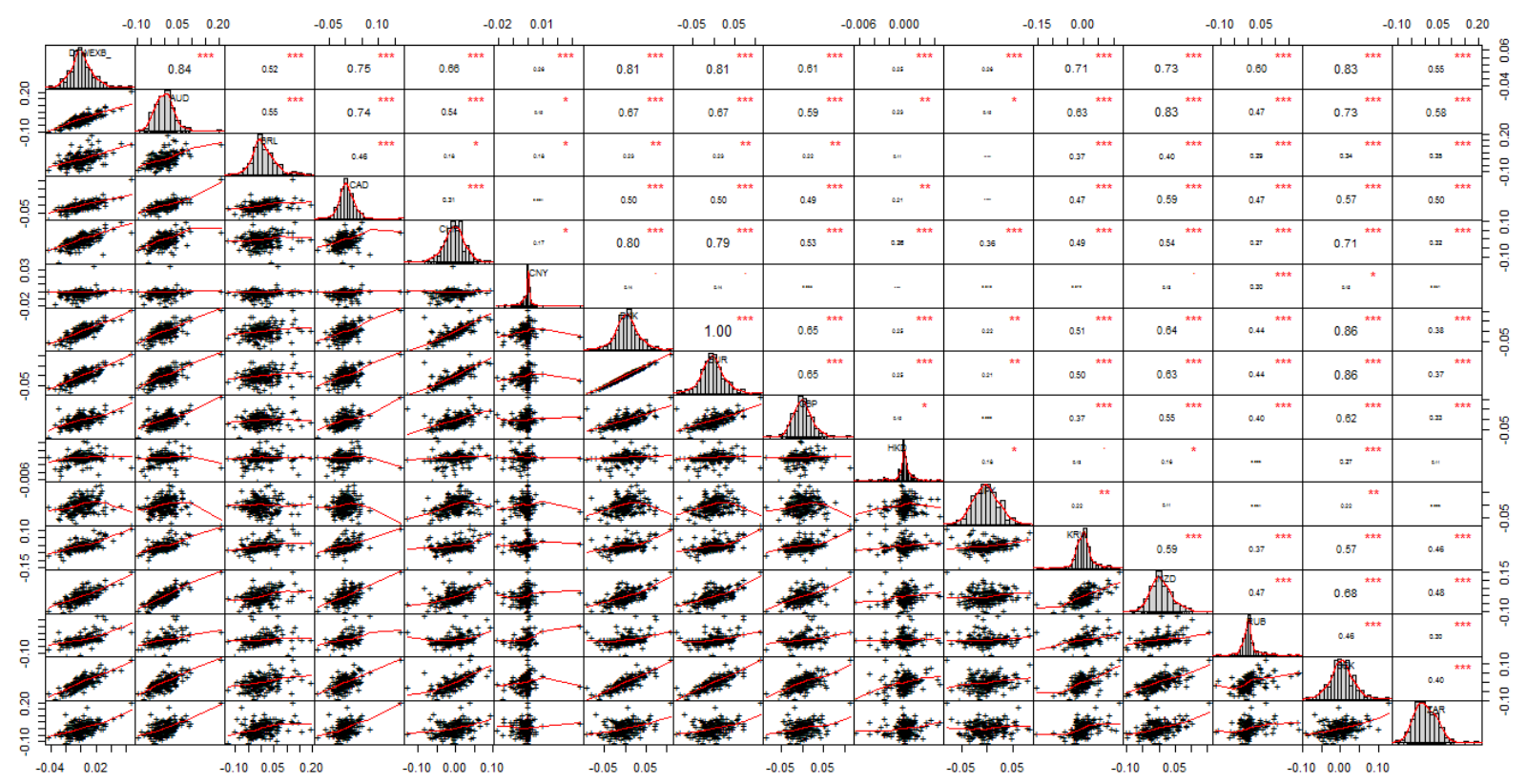

Figure 1. Return distribution comparison of the 15 currencies and the US Dollar Index (DTWEXB)

Figure 2 also shows that all currency $\log$ returns display positive correlation with the US Dollar Index (DTWEXB). 
Return Distribution Comparison

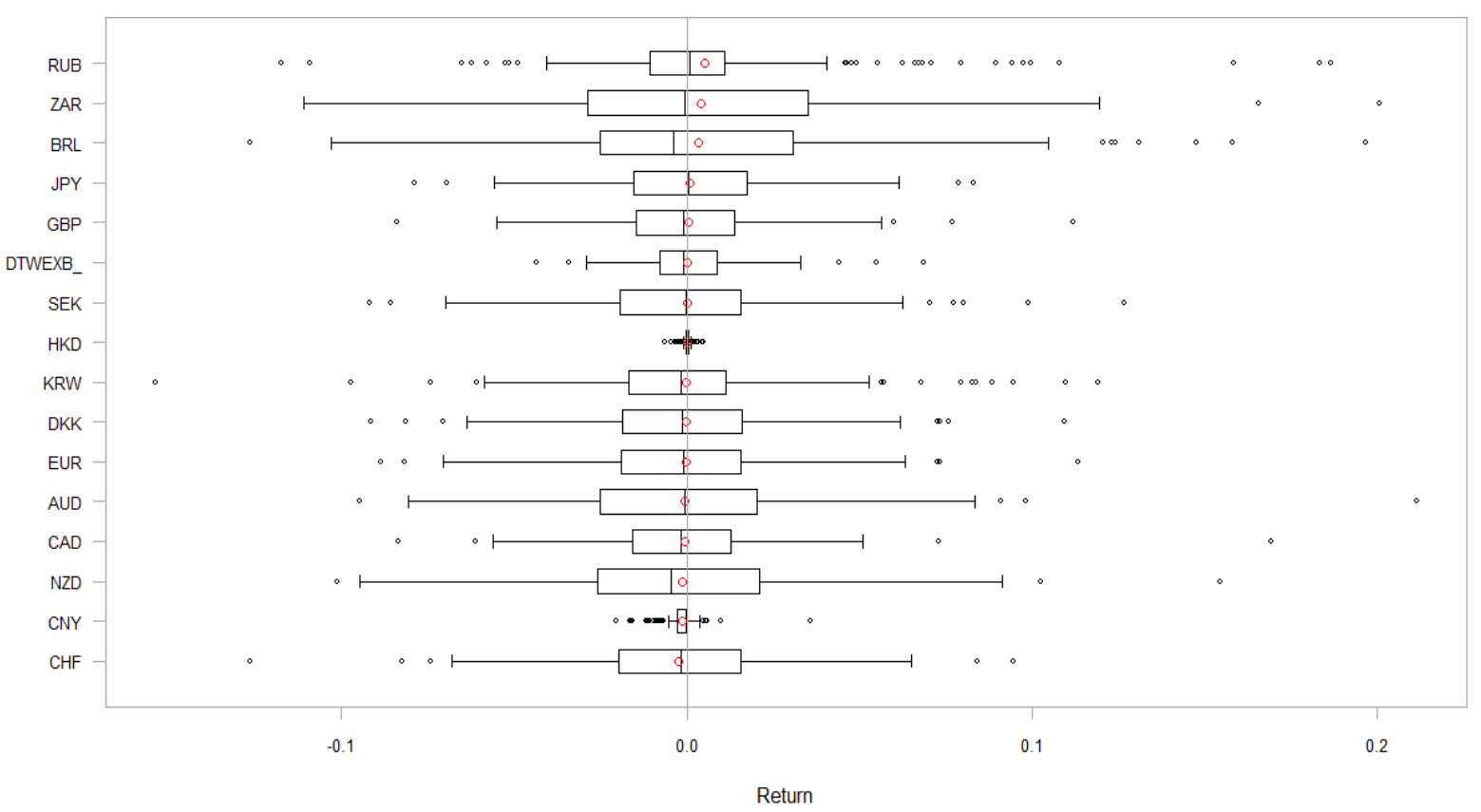

Figure 2. Correlation analysis and return distribution comparison of the 15 currencies and the US Dollar Index (DTWEXB)

\subsection{Performance of Portfolio Allocation Strategies}

To generate a holistic assessment of our findings, recourse is made towards the averages of 8 months out-of-sample results obtained under each of the seven strategies deployed to compose the portfolio of 15 currencies. The corresponding results are displayed in Table 2.

Table 2. Averages of 8 months out-of-sample results obtained under each of the 7 strategies deployed

\begin{tabular}{|c|c|c|c|c|c|c|c|}
\hline AVERAGES & $\begin{array}{c}\text { Expected } \\
\text { Return }\end{array}$ & $\begin{array}{c}\text { Std. } \\
\text { Deviation }\end{array}$ & $\begin{array}{c}\text { Beta } \\
\text { Coeff. }\end{array}$ & $\begin{array}{c}\text { Sharpe } \\
\text { Ratio }\end{array}$ & $\begin{array}{c}\text { Jensen's } \\
\text { alpha }\end{array}$ & $\begin{array}{c}\text { Treynor } \\
\text { Ratio }\end{array}$ & $\begin{array}{c}\text { Roy } \\
\text { Ratio }\end{array}$ \\
\hline Equal Allocation & $0.0184 \%$ & $2.0567 \%$ & 1.2551 & 0.0090 & $0.0201 \%$ & $0.0147 \%$ & $0.9625 \%$ \\
\hline Single index (Short-Selling) & $0.1707 \%$ & $0.3394 \%$ & -1.3145 & 0.5030 & $0.1691 \%$ & $-0.1300 \%$ & $50.6841 \%$ \\
\hline Single index (No Short-Selling) & $0.4174 \%$ & $3.1093 \%$ & 1.4161 & 0.1340 & $0.4192 \%$ & $0.2951 \%$ & $13.4452 \%$ \\
\hline Tangency (Gaussian) & $0.2178 \%$ & $0.3956 \%$ & -0.0117 & 0.5503 & $0.2178 \%$ & $-19.9491 \%$ & $55.3429 \%$ \\
\hline Tangency (Skewed Student-t) & $0.3335 \%$ & $0.6366 \%$ & -0.0758 & 0.5235 & $0.3334 \%$ & $-4.4336 \%$ & $52.5375 \%$ \\
\hline Target $(0.5 \%)$ (Gaussian) & $0.5000 \%$ & $0.9908 \%$ & -0.0120 & 0.5048 & $0.4999 \%$ & $17.1299 \%$ & $50.6316 \%$ \\
\hline Target $(0.5 \%) \quad$ (Skewed Student- $t)$ & $0.5022 \%$ & $1.0034 \%$ & -0.1184 & 0.5008 & $0.5019 \%$ & $-4.2680 \%$ & $50.2296 \%$ \\
\hline
\end{tabular}

\subsubsection{Expected Return}

Under expected return analysis, it transpires that targeted return strategy under student t-distribution unleashed the best return while equal allocation registered the worst performance. However, investors rarely cling to expected returns when gauging on the type of portfolio strategy to deplore. The underlying rationale is that expected return does not factor in risk but simply projects a desired rate of return. Alternatively stated, it is clear that imposing a certain level of return on the portfolio will automatically trigger such imposed return as to beat other strategies deployed. However, assessing the other side of the coin, such strategy does not factor in the level of risk embodied in that higher return relates to higher risk so much so that at any point in time, the portfolio can significantly underperform vis-à-vis an equally allocated portfolio. Such a finding also shows that $0.5 \%$ return per month constitutes a highly optimistic approach to return. Technically speaking, the superior return generated by skewed student-t distribution is due to the fact that financial data are usually non-normal in practice so that the use of normal distribution underperforms that of the skewed student-t distribution. This, despite the fact that 
both fall under the purview of targeted return strategy. The worst performance noted in the case of equal allocation can be accounted for by the fact that the portfolio is passive to changes in returns and thus fails to ride through the different phases of the market trends in the portfolio of currencies.

\subsubsection{Standard Deviation}

Under pure risk analysis via standard deviations without factoring in any element of return, findings show that the single-index strategy with short sales generates the best result. Ironically, the worst performance was also triggered under the single-index strategy but under no short sales being incorporated. Such a result implies that the very existence of short sales in a portfolio enables to trigger lower risk in terms of standard deviation. Most importantly, the distinctive feature of short sales significantly alters the single-index strategy in the sense that short sales are introduced as a dynamic feature in our portfolio modelling exercise in view of harnessing maximum benefits from asset holdings. Indeed, as per our short sales formula, it can be noted that only currencies which trigger returns beyond a threshold level are retained in the portfolio. The equal allocation strategy did not also fare up well under standard deviation analysis. Interestingly, it is found that tangency portfolio, under both student t-distribution and Gaussian approach, emerged as the second and third best performers out of the various strategies under consideration. Such a result bodes well with the fact that under tangency analysis, the whole aim is geared towards triggering low risk and high return. Targeted return fares poorly on the back of no due consideration being attribute to the other side of return, that is risk.

\subsubsection{Beta}

Beta, deemed as the systematic component of any risk imbued in portfolio analysis, represents one of our key performance indicators. Only equal allocation and single-indexed model without short sales post positive returns. All other strategies unleash negative returns. Technically speaking, betas can be zero, above one or below 1, but this holds in equity markets. In the current context, betas can take different values. Equal allocation and single-indexed strategy without short sales, engender aggressive betas. This means that should the benchmark return generate a return of $10 \%$, then, both strategies will outperform the benchmark return. All other strategies post negative betas, that is betas which go against the movement in the market. If the market triggers positive returns, the strategy will actually register negative returns. Conversely, in the case that the market exhibits negative returns, all the other strategies (excluding equal allocation and single-indexed strategy) will post positive returns with single-indexed model under short sales unleashing the best performance. Thus, short sales can have substantial impact on the performance of a portfolio. Such a state of affairs implies that short sales can be particularly beneficial during crisis times as witnessed during the US subprime crisis. It is important to note that all those strategies which register positive betas, basically pertain to passive strategies which do not take into account the evolution of the market index. For instance, the equal allocation strategy constitutes weights irrespective of changes in returns. In the same vein, the single-indexed strategy without short sales does not factor in the changes in market returns.

\subsubsection{Sharpe Ratio}

Sharpe ratio takes into account the risk component of a portfolio and is thereby a better analytical tool to gauge on the superiority of an adopted strategy. Out of the various strategies considered, it transpires that the tangency portfolio under Gaussian and student t-distribution came out as the first and second best performers respectively. Consequently, it can be conjectured that the tangency portfolios constitute interesting optimizing points for investors too. The equal allocation strategy manifested as the worst performer. This glaringly shows that equal allocation is not really helpful as it does not cater for the distinct types of asset distributions. Above all, in the current context, for the same asset type (currency), it is most likely that equal allocation is unlikely to unleash superior returns as it fails to ride through the distinct phases of market trends.

\subsubsection{Jensen's Alpha}

Technically speaking, the Jensen's performance is measured as the expected return of the portfolio minus the expected return of the portfolio à la CAPM approach. In this case, the whole term collapses into expected portfolio return (which is nothing else but our first metric of assessment used) minus the product of beta and that of the market return. Therefore, since market return is exogenous by nature and beta is computed based on the various strategies, expected portfolio return has a strong say when it comes to assessing the performance of portfolio under Jensen's alpha. This fact explains the reason behind the top positions noted in the case of targeted return strategies. Findings actually show exactly the same ranking of portfolios on the basis of the two performance indicators, expected return and Jensen's alpha. Besides, it is noteworthy to say that skewed student-t distribution generates the best performance within targeted return strategies. 


\subsubsection{Treynor Ratio}

Reflecting the risk-adjusted version of return based on systematic risk, the Treynor ratio constitutes a widely coveted tool among investment professionals. Findings show that the targeted return based on Gaussian distribution triggered the best performance while the tangency portfolio based on Gaussian returns unleashed the worst performance. Intriguingly, strong negative returns are noted in the case of the tangency portfolio based on Gaussian distribution. This could be feasibly attributed to the presence of outliers.

\subsubsection{Roy Ratio}

The Roy ratio shows that the tangency portfolio strategies generate the best performances with quite similar results to those observed under the Sharpe ratio analysis. Such a finding shows the feasible link between Sharpe ratio and Roy ratio.

\subsubsection{Value-at-Risk}

Perceived as one of the core metrics for risk management, Value-at-Risk is also given due consideration in our analysis. The five different VaR metrics are calculated based on a monthly horizon at a 5\% significance level.

To ease the comparative analysis on the basis of the VaR performance indicator, an average for the 5 VaRs is calculated for each strategy. The results are presented in Table 3. The findings show that the tangency portfolio under Gaussian distribution followed by its homologue under skewed student-t distribution emerge as the best performers on the back of lowest negative return. The worst performers consist of the single index model with and without short sales. Hence, the tangency approach for portfolio evaluation is found to be particularly helpful under VaR assessment. Ironically, in the case of imposed returns, Value-at-Risk does not fare better. It is noteworthy to point out that the performance analysis undertaken on the basis of the 'average' value is in accordance with an analysis on the basis of the individual VaRs.

Table 3. Performance of the strategies on the basis of the 5 value-at-risk measures

\begin{tabular}{lcccccc}
\hline & \multicolumn{5}{c}{ Value-at-Risk (VaR) } \\
\cline { 2 - 7 } & $\begin{array}{c}\text { Parametric Gaussian } \\
\text { (Eq-weight) }\end{array}$ & $\begin{array}{c}\text { Historical } \\
(\text { Eq-weight) }\end{array}$ & $\begin{array}{c}\text { Historical } \\
\text { (Boots-trapping) }\end{array}$ & $\begin{array}{c}\text { Monte-Carlo } \\
\text { Simulation }\end{array}$ & $\begin{array}{c}\text { Parametric GHD AVERAGE } \\
\text { (Eq-weight) }\end{array}$ & VaR \\
\hline Equal Allocation & -0.00301 & -0.00241 & -0.00395 & -0.00259 & -0.00234 & -0.00286 \\
Single index (Short-Selling) & -0.00526 & -0.00571 & -0.00947 & -0.00561 & -0.00596 & -0.00640 \\
Single index (No Short-Selling) & -0.01820 & -0.00684 & -0.00387 & -0.01629 & -0.01246 & -0.01153 \\
Tangency (Gaussian) & -0.00186 & -0.00109 & -0.00080 & -0.00177 & -0.00144 & -0.00139 \\
Tangency (Skewed Student- $t$ ) & -0.00301 & -0.00170 & -0.00142 & -0.00284 & -0.00231 & -0.00225 \\
Target (0.5\%) (Gaussian) & -0.00437 & -0.00229 & -0.00170 & -0.00414 & -0.00331 & -0.00316 \\
Target $(0.5 \%)$ (Skewed Student- $t$ ) & -0.00458 & -0.00236 & -0.00212 & -0.00431 & -0.00346 & -0.00337 \\
\hline
\end{tabular}

\section{Conclusions}

The study implements the passive "naïve" strategy and 6 active portfolio optimization strategies on a basket of 15 currencies with US dollar as the base currency for the period spanning from 1999 to 2015. The comparative analysis is done on the basis of specific portfolio performance indicators. Overall, the results show that all the active allocation techniques outperformed the passive strategy. Furthermore, the study reveals that both tangency portfolios, with the Gaussian or the skewed student-t distribution perform best, particularly on the basis of highest Sharpe reward-to-variability ratio and lowest Value-at-Risk. The corresponding low magnitude beta coefficients means that they are conservative towards fluctuations in the exchange market. An extension to this study could be investigating the performance of tangency portfolios using alternative risk metrics to the standard deviation, such as the Value-at-Risk or the Expected Shortfall.

\section{References}

Black, F., \& Litterman, R. (1992). Global Portfolio Optimization. Financial Analysts Journal, 48(5), 28-43. http://dx.doi.org/10.2469/faj.v48.n5.28

Borch, K., Hester, D., \& Tobin, J. (1969). Risk Aversion and Portfolio Choice. Econometrica, 37(1), 162. http://dx.doi.org/10.2307/1909223

Caporin, M., \& Lisi, F. (2011). Comparing and Selecting Performance Measures for Ranking Assets. SSRN Electronic Journal. http://dx.doi.org/10.2139/ssrn.1859869 
Crama, Y., \& Schyns, M. (2003). Simulated annealing for complex portfolio selection problems. European Journal of Operational Research, 150(3), 546-571. http://dx.doi.org/10.1016/s0377-2217(02)00784-1

Daly, J., Crane, M., \& Ruskin, H. (2008). Random matrix theory filters in portfolio optimisation: A stability and risk assessment. Physica A: Statistical Mechanics And Its Applications, 387(16-17), 4248-4260. http://dx.doi.org/10.1016/j.physa.2008.02.045

Hochreiter, R. (2008). Evolutionary Stochastic Portfolio Optimization. Natural Computing in Computational Finance. http://dx.doi.org/10.1007/978-3-540-77477-8_5

Koh, Y., \& Lee, S. (2011). Cross-listing effect of U.S. casino companies: Risk-adjusted performances. International Journal of Hospitality Management, 30(4), 1055-1058. http://dx.doi.org/10.1016/j.ijhm.2011.03.003

Markowitz, H. (1952). Portfolio Selection. The Journal of Finance, 7(1), 77. http://dx.doi.org/10.1111/j.1540-6261.1952.tb01525.x

Mishra, S. K., Panda, G., \& Meher, S. (2009). Multi-objective Particle Swarm Optimization Approach to Portfolio Optimization. In World Congress on Nature and Biologically Inspired Computing. http://dx.doi.org/10.1109/nabic.2009.5393659

Parpas, P., \& Rustem, B. (2007). Computational Assessment of Nested Benders and Augmented Lagrangian Decomposition for Mean-Variance Multistage Stochastic Problems. INFORMS Journal on Computing, 19(2), 239-247. http://dx.doi.org/10.1287/ijoc.1050.0163

Sereda, E., Bronshtein, E., Rachev, S., Fabozzi, F., Wei, S., \& Stoyanov, S. (2010). Distortion risk measures in portfolio optimization.

Sharpe, W. (1963). A Simplified Model for Portfolio Analysis. Management Science, 9(2), 277-293. http://dx.doi.org/10.1287/mnsc.9.2.277

Sharpe, W. (1964). Capital Asset Prices: A Theory of Market Equilibrium under Conditions of Risk. The Journal Of Finance, 19(3), 425. http://dx.doi.org/10.1111/j.1540-6261.1964.tb02865.x

Steiner, M., \& Wittkemper, H. (1997). Portfolio optimization with a neural network implementation of the coherent market hypothesis. European Journal of Operational Research, 100(1), 27-40. http://dx.doi.org/10.1016/s0377-2217(95)00339-8

Troha, M. (2011). Portfolio Optimization as Stochastic Programming with Polynomial Decision Rules. PhD thesis, Department of Computing, Imperial College London, September 2011.

Von Neumann, J., \& Morgenstern, O. (1953). Theory of games and economic behavior. Princeton: Princeton University Press.

Zimmermann, H., Neuneier, R., \& Grothmann, R. (2001). Multi-agent modeling of multiple FX-markets by neural networks. IEEE Trans. Neural Netw., 12(4), 735-743. http://dx.doi.org/10.1109/72.935087

\section{Copyrights}

Copyright for this article is retained by the author(s), with first publication rights granted to the journal.

This is an open-access article distributed under the terms and conditions of the Creative Commons Attribution license (http://creativecommons.org/licenses/by/4.0/). 